\title{
Gas-Forming Intrahepatic Abscess: A Possible Complication of Arterial Infusion Chemotherapy
}

\author{
Carl J. D’Orsi, ${ }^{1}$ William Ensminger, ${ }^{2}$ Edward H. Smith, ${ }^{1}$ and Michael Lew ${ }^{3}$ \\ Department of ${ }^{1}$ Radiology and ${ }^{3}$ Division of Clinical Microbiology, Sidney Farber Cancer Institute, Harvard Medical School, \\ Boston, Massachusetts USA; and ${ }^{2}$ Department of Medicine, University of Michigan, Ann Arbor, Michigan USA
}

\begin{abstract}
Three cases of intrahepatic gas-forming abscesses are presented with liver metastases and indwelling hepatic artery catheters for drug infusion. This may represent a further complication of intrahepatic arterial drug infusion. Possible etiologies along with future prospects for this form of therapy are discussed.
\end{abstract}

Key words: Abscess, gas-forming - Liver - Clostridium - Metastases.

Infusion of chemotherapeutic agents via selective catheterization can deliver high doses of drug to a selective area in either primary or secondary malignancy. The technique has been utilized most frequently for treatment of liver metastases from gastrointestinal primaries utilizing 5-fluorouracil (5-FU) and 5-fluoro2-deoxyuridine (5-FUDR) [1-3]. Despite improved therapeutic results, this form of therapy is associated with the potential for serious complications, including infection and arterial thrombosis [4]. We wish to report 3 cases of intrahepatic gas-forming abscess which developed after hepatic arterial chemotherapy.

\section{Case Reports}

Case 1

A 52-year-old white female was well until 2 months prior to her first admission to the Sidney Farber Cancer Institute (SFCI), when she experienced nausea, vomiting, constipation, and blood-tinged diarrhea. Physical examination revealed massive hepatomegaly extending $16 \mathrm{~cm}$ below the right costal margin. Intravenous pyelogram, barium enema, and sigmoidoscopy were nondiagnostic.

Address reprint reguests to: Carl J. D'Orsi, M.D. Department of Radiology, Peter Bent Brigham Hospital, Boston, MA 02115, USA
However, both ultrasound and radionuclide examination of the liver revealed multiple focal defects. Subsequent liver biopsy showed adenocarcinoma consistent with a gastrointestinal primary.

After the patient was admitted to SFCI, a hepatic artery catheter was placed percutaneously via the left brachial artery and gastroduodenal artery was occluded with Gelfoam. She was started on 5-FUDR with constant infusion. After 4 weeks, the patient developed epigastric pain related to the infusion, and angiography revealed a thrombosed hepatic artery. At this point, the catheter was removed and she was treated with weekly intravenous doses of 5-FU. Two months after the catheter was removed, the hepatic artery was still occluded. The patient remained stable for 4 months after the catheter removal when she developed the sudden onset of fever and chills. She was admitted to SFCI and found to be febrile to $102^{\circ} \mathrm{F}$, hypotensive with a systolic blood pressure of $60 \mathrm{~mm}$ of $\mathrm{Hg}$, and tachycardic. An anteroposterior (AP) radiograph of the abdomen at this time was within normal limits (Fig. 1 A). Blood cultures were obtained and she was then empirically started on gentamicin, clindamycin, and Keflex because of a known penicillin allergy. A second abdominal film 2 days after the first film revealed several round gas collections over the liver (Fig. 1B), compatible with gas-containing pyogenic hepatic abscesses. Blood cultures were positive for Clostridium septicum. She was maintained on intravenous (i.v.) antibiotics for 2 weeks and subsequent blood cultures were negative. She was then placed on oral chloramphenicol, and although still intermittently febrile to $101^{\circ} \mathrm{F}$, clinically felt better. Gas-containing processes were still present on abdominal films. The patient was discharged on oral chloramphenicol to be followed by her local physician. She developed a progressive anorexia and 6 weeks after discharge died at home.

\section{Case 2}

A 51-year-old white female had a 15-year history of a "scalp cyst" which changed in size and color 1 year prior to admission. Three months later, biopsy of the scalp lesion along with a needle biopsy of a right pulmonary nodule revealed spindle cell malignant melanoma. The patient was asymptomatic for 5 months, but then experienced anorexia, nausea, weight loss, fatigue, and intermittent low-grade fever. She was found to have massive hepatomegaly on physical examination with a positive liver and bone scan at this time and was admitted to the hospital for a course of dimethyltriazeno-imidazole-carboximide (DCTI), vincristine (VCR), and Cytoxan (CTX). Her disease progressed rapidly and she was admitted to SFCI in a preterminal state. An AP view of the abdomen (Fig. 2A) was obtained on admission. Despite her preterminal 

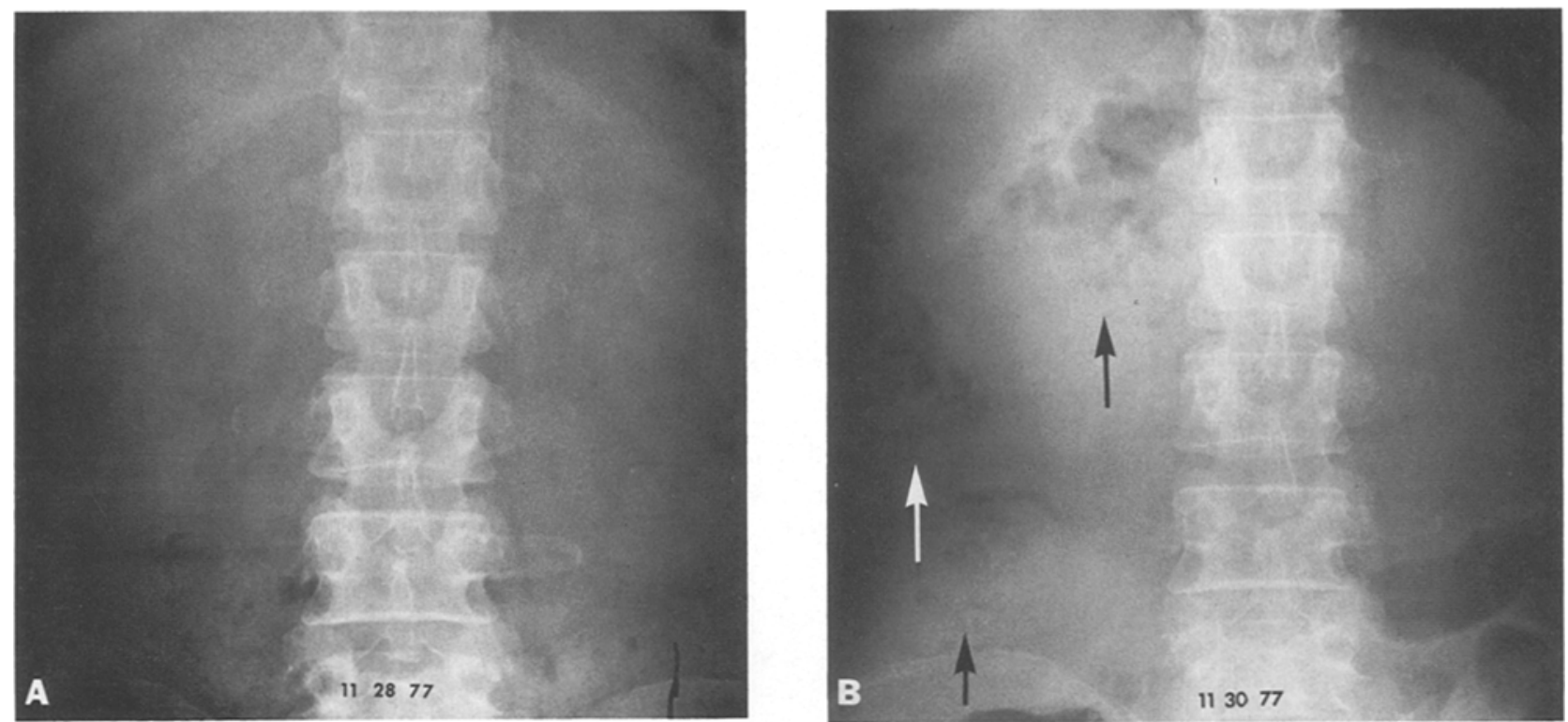

Fig. $1 \mathrm{~A}$ and B. AP abdominal radiograph. A No evidence for gas in the right upper quadrant. B Several ill-defined, gas-containing areas (arrows) have developed over a 2 day interval
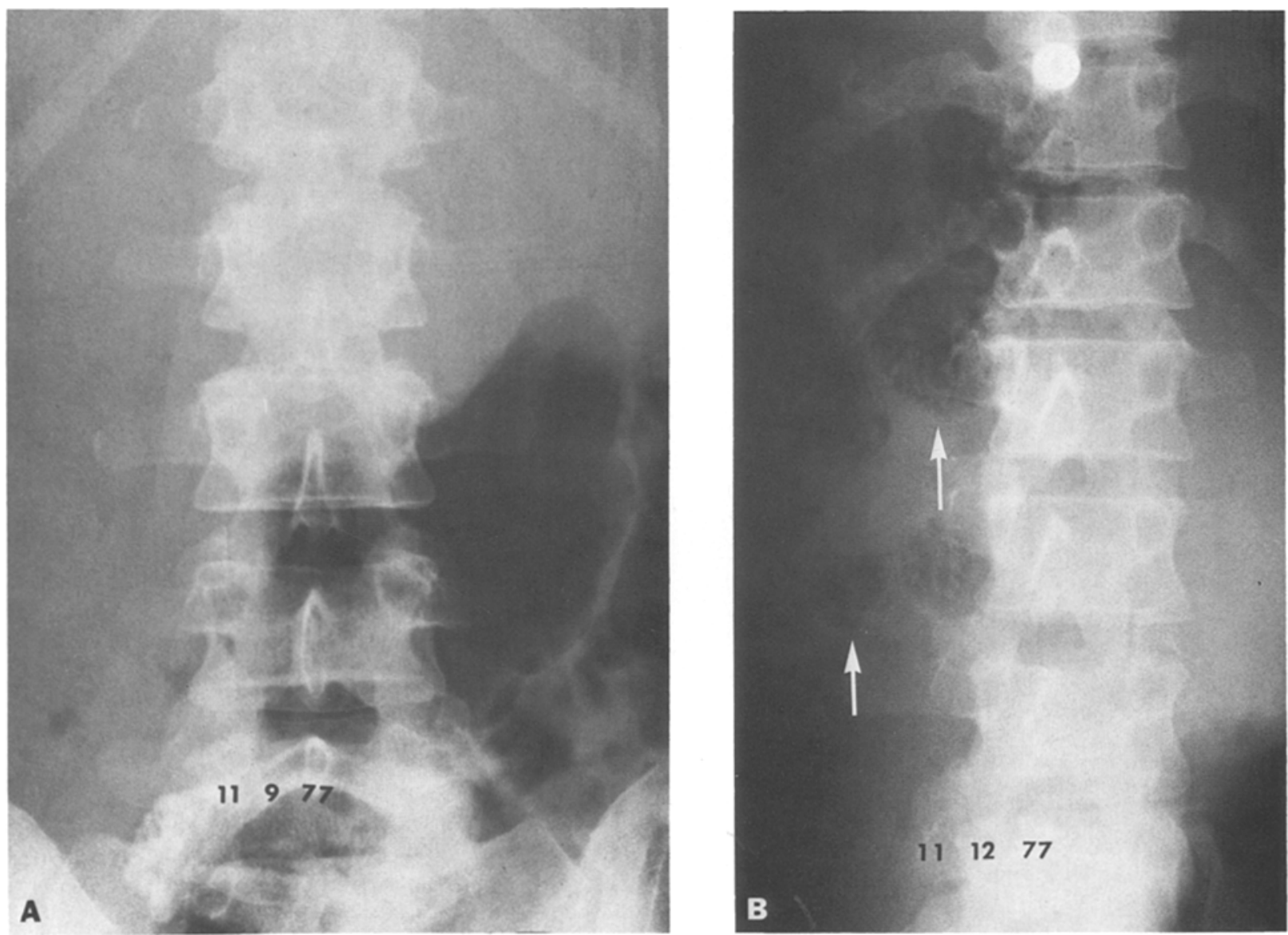

Fig. 2 A and B. Initial AP abdominal radiograph. A Gas within stomach and large bowel. B Follow-up examination. Mottled gas densities 3 days later in the area of the right lobe of the liver (arrows) 

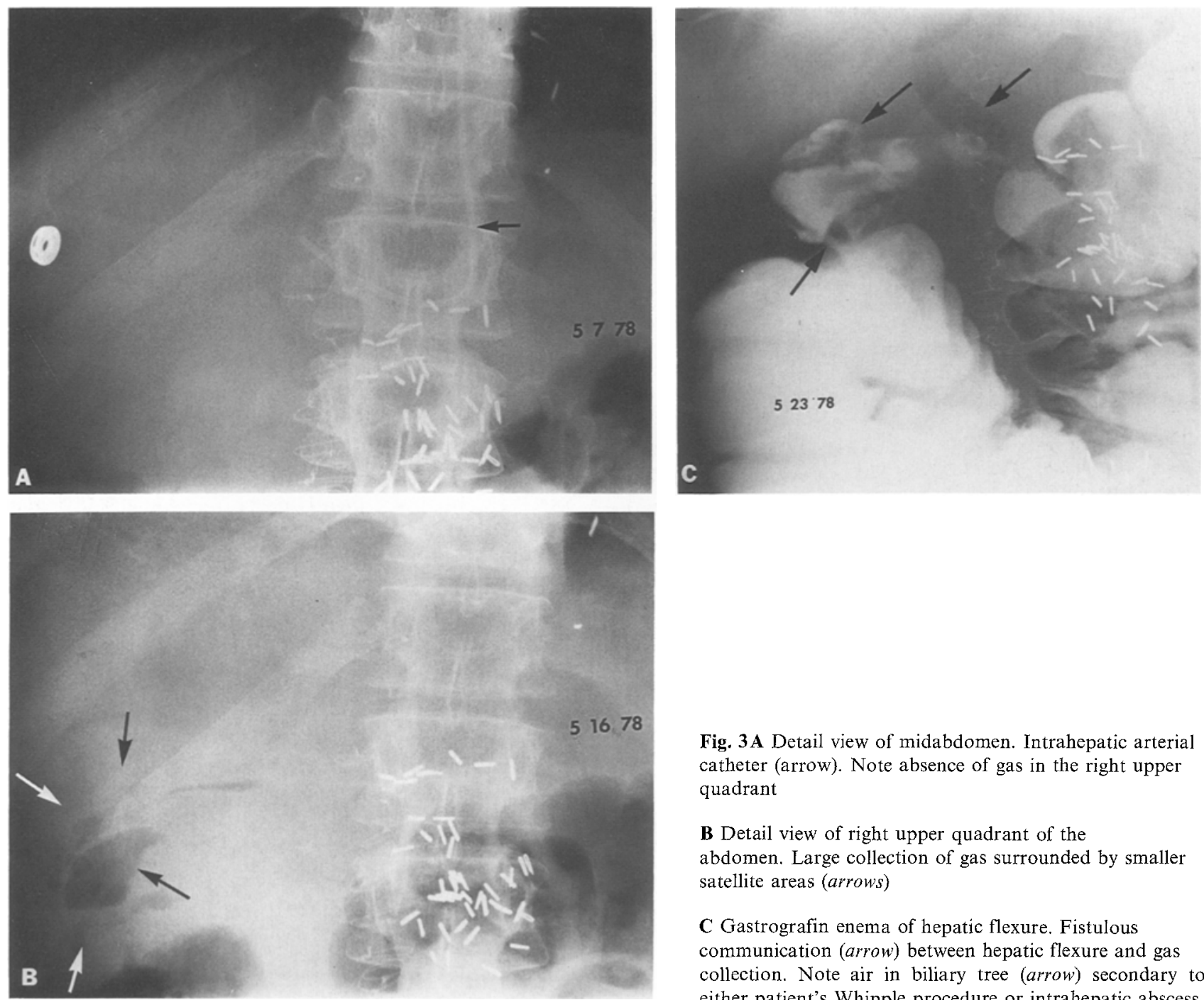

Fig. 3 A Detail view of midabdomen. Intrahepatic arterial catheter (arrow). Note absence of gas in the right upper quadrant

B Detail view of right upper quadrant of the abdomen. Large collection of gas surrounded by smaller satellite areas (arrows)

C Gastrografin enema of hepatic flexure. Fistulous communication (arrow) between hepatic flexure and gas collection. Note air in biliary tree (arrow) secondary to either patient's Whipple procedure or intrahepatic abscess

state, the patient and family desired further therapy and on the day following admission, an hepatic arterial catheter was placed for infusion chemotherapy. The gastroduodenal artery was occluded in an attempt to diminish toxicity. A $1 \mathrm{hr}$. infusion of 1,3bis(2-chlorethyl)-1-nitrosourea (BCNU) was administered without incident. Ten hours after the drug infusion, the patient developed a fever of $103^{\circ} \mathrm{F}$. Blood and urine cultures taken at that time were subsequently negative. She was immediately started on oxacillin and gentamicin. Within $12 \mathrm{~h}$ of initiation of antibiotic therapy, the patient became afebrile and felt much improved. Thirty hours after the BCNU infusion, fever again developed, with associated hypotension. The hematocrit rapidly fell from $35 \%$ to $20.8 \%$ with the development of brown serum and urine. An abdominal film at this time (Fig. 2B) showed multiple loculated gas collections in the right upper quadrant compatible with gas-forming hepatic abscesses. Two sets of blood cultures were obtained which subsequently grew Closiridium ramosum. Despite intensive therapy, the patient died $10 \mathrm{hr}$. later, $40 \mathrm{hr}$. post BCNU infusion.

\section{Case 3}

A 29 -year-old white male presented locally complaining of recurring right upper quadrant pain of 5 to 6 months', duration. Diagnos- tic work-up revealed obstructive jaundice. Laparotomy revealed a fungating lesion of the ampulla of Vater, which proved to be moderately well-differentiated adenocarcinoma. No definitive surgery was done and the patient was transferred to SFCI. A Whipple procedure was performed and at pathology the tumor was deep to the duodenal epithelium in the region of the junction of the common bile duct and major pancreatic duct. Lymph channels involved with tumor as well as superficial implants on the head of the pancreas were noted. In view of this, aggressive radio- and chemotherapy were planned. A liver scan in mid September 1977 was normal. In December 1977 , after completing 4,450 rads to the upper abdomen and para-aortic nodes, the patient was begun on intravenous 5-FU therapy at $500 \mathrm{mg}$ for 1 week. During treatment, the patient's CEA rose from $19 \mathrm{mg} / \mathrm{ml}$ in December 1977 to $99 \mathrm{mg} / \mathrm{ml}$ in February 1978. A liver scan done on March 30 , 1978, revealed a large liver with a focal defect in the right lobe. In April 1978, chemotherapy was escalated to $1,500 \mathrm{mg}$ i.v. of 5-FU and the CEA rose to $220 \mathrm{mg} / \mathrm{ml}$.

He was admitted to SFCl on March 3, 1978, for placement of an hepatic artery catheter. The gastroduodenal artery was not identified and presumed to have been ligated during prior surgery. Infusion of $5 \mathrm{mg}$ of dichloromethotrexate was then begun. Due to persistent abdominal pain, a second catheter was positioned 
further peripherally (Fig. 3A). Nine days later the patient became febrile to $104.5^{\circ} \mathrm{F}$ and blood cultures at this time grew Clostridium ramosum and Clostridium sporogenes.

An AP abdominal film (Fig. 3B) revealed intrahepatic gas in the biliary tree and an amorphous gas collection over the lower lobe of the liver. The patient was started on i.v. oxacillin, Chloromycetin, and gentamicin but maintained a temperature of $101^{\circ}$ to $102^{\circ} \mathrm{F}$. In an effort to further localize the right upper quadrant process prior to surgery, we gave a Gastrografin enema, and a fistulous communication between the right hepatic flexure and the amorphous gas collection was demonstrated (Fig. 3C). At surgery, a fluid collection adherent to the liver and hepatic flexure with fistulous communication between them was seen. Culture of the fluid failed to grow either aerobic or anaerobic bacteria. The patient was discharged having failed all available forms of therapy for progressive liver metastases and died 2 weeks later.

\section{Discussion}

Mortality for pyogenic liver abscess can range from $68 \%$ to $88 \%$, with a more recent figure of about $50 \%$ being reported [5]. In part, this high mortality appears to be related to a delay in diagnosis [6]. Ochsner et al. [7] in an extensive 10-year review, found that liver abscesses were primarily a complication of intra-abdominal suppurative processes associated with antecedent lesions in the portal area. Appendicitis was the etiologic agent in $34.2 \%$. An update of hepatic abscesses by Sherman and Robbins in 1958 [8] revealed the most frequent antecedent cause to be cholangitis secondary to obstruction of the biliary tree. Recent reviews stress the importance of anaerobic organisms as a cause of intrahepatic abscess [9, 10]. The presence of mottled gas in the right upper quadrant overlying the liver allows the radiologist to offer a differential diagnosis which includes gascontaining intrahepatic pyogenic abscess.

The association of hepatic metastases and gasproducing abscess is not a common one. Kahn et al. [11] describe a Clostridium hepatic abscess with a metastatic lesion at its center. Clouse et al. [4], in a review of 75 patients representative of 127 transbrachial hepatic artery catheterizations for drug infusion, did not report hepatic abscess as a complication. The most frequent major complication was partial or complete arterial thrombosis. However, four patients did develop infection at the arteriotomy site.

The patients presented here had in common the introduction of an intrahepatic arterial catheter for therapeutic purposes, Clostridium septicemia, and intrahepatic gas-producing abscess. The time from introduction of the hepatic catheter to demonstration of Clostridium septicemia and hepatic abscess, however, varied significantly from 1 day in case 2, 9 days in case 3 , and 4 months in case 1 . We reviewed all our patients with Clostridium septicemia for the past 2 years and found a total of four patients, three of whom are presented herein and had hepatic arterial indwelling catheters. Thus the presence of an indwelling hepatic artery catheter does appear to be significant in our patients for production of both Clostridium sepsis and hepatic abscess.

There are experimental data to show that intravenous administration of Clostridium spores into mice with various tumors promoted development of toxinproducing cells in tumor tissue, whereas mice without tumors were unaffected [12]. Trump et al. [13] suggest that the hypoxic milieu of tumor tissue may be conducive to the germination of Clostridium spores and also provides a medium for colonization of other anaerobes. Reports of ligation of the hepatic artery with subsequent development of gas-containing hepatic abscess or Clostridium infection support the importance of hypoxia $[14,15]$. It is interesting to speculate from these data whether the thrombotic occlusion of the hepatic artery as a complication of hepatic artery catheterization in case 1 and the presence of an indwelling hepatic catheter in cases 2 and 3 provided a favorable hypoxic background for the ultimate growth of Clostridium. Clostridium septicemia has been reported in association with malignant disease, but it appears that damaged tissue is needed as a background prior to growth $[16,17]$. A recent report indicates an increased survival of patients with liver metastases when dearterialization of the liver coupled with intraportal infusion chemotherapy is done instead of arterial infusion alone [18]. If this technique is to be used, one wonders if the incidence of Clostridium sepsis will increase.

The presence of a mottled gas pattern in the right upper quadrant in a setting of liver metastases and an indwelling hepatic artery catheter should strongly raise the possibility of hepatic abscess and may represent a further complication of intra-arterial hepatic infusion in the immunosuppressed patient with liver metastases.

Acknowledgement. This work was supported in part by USPHS Grant GM 18674.

\section{References}

1. Cady B, Oberfield R: Regional infusion chemotherapy of hepatic metastases from carcinoma of the colon. Am J Surg 127:220-227, 1974

2. Ansfield T, Ramirez Gi, Davis H, Wirtanen GW, Johnson RO, Bryan GT, Manalo FB, Borden EC, Davis TE, Esmaili M: Further clinical studies with intrahepatic arterial infusion with 5 fluorouracil. Cancer 36: 2413-2417, 1975 
3. Ramming KP, Sparks FC, Eilber FR, Holmes EC, Morton DL: Hepatic artery ligation and 5-fluorouracil infusion for metastatic colon carcinoma and primary hepatoma. Am J Surg 132: 236-242, 1976

4. Clouse M, Ahmed R, Ryan RB, Oberfield RA, McCaffrey JA: Complications of long term transbrachial hepatic arterial infusion chemotherapy. Am J Roent 129:799-803, 1977

5. Madayag MA, Lefleur RS, Braunstein P, Beranbaum E, Bosniak M: Radiology of hepatic abscess. NY State J Med 75: 1417-1423, 1975

6. Lee JF, Block GE: Changing clinical pattern of hepatic abscesses. Arch Surg 104: 465-470, 1972

7. Ochsner A, DeBakey M, Murray S: Pyogenic abscess of the liver: an analysis of forty-seven cases with review of the literature. Am J Surg 40: 292-319, 1938

8. Sherman J, Robbins SL: Changing trends in casuistics of hepatic abscess. Am J Med 28: 943-950, 1960

9. Gorbach SL, Bartlett JG: Anaerobic infection (Part 1). N Engl $J$ Med 290: 1177-1183, 1974

10. Sabbaj J, Sutler VL, Finegold SM: Anaerobic pyogenic liver abscess. Ann Int Med 77:629-638, 1972

11. Kahn SP, Lindenauer SM, Wojtalk RS, Hildreth D: Clostridium hepatic abscess. Arch Surg 104: 209-212, 1972
12. Malmgren RA, Flanigan CC: Localization of the vegetative form of Clostridium tetani in mouse tumors following intravenous spore administration. Cancer Res 15: 473-478, 1955

13. Trump DL, Fahnestock R, Cloutier CT, Dickman MD: Anaerobic liver abscess and intrahepatic metastases. Cancer 41:682685,1978

14. Thorley LG, Figiel LS, Figiel SJ, Rush DK: Roentgenographic findings in accidental ligation of the hepatic artery: a case report. Radiology 85:56-58, 1965

15. Chau AYS, Goldbloom VC, Gurd FN: Clostridial infection as a cause of death after ligation of the hepatic artery. Arch Surg 63: 390-402, 1951

16. Cabrera A, Tsukada Y, Pickren JW: Clostridium gas gangrene and septicemia in malignant disease. Cancer 18: 800-806, 1965

17. Boggs DR, Frei E III, Thomas LB: Clostridial gas gangrene and septicemia in four patients with leukemia. $N$ Engl $J \mathrm{Med}$ 259: 1255-1258, 1958

18. Nagasue N, Inokucki K, Koboyski M, Ogawa Y, Iwaki A, Yukaya $\mathrm{H}$ : Hepatic dearterialization for nonresectable primary and secondary tumors of the liver. Cancer 38: 2593-2603,1976

Received: July 21, 1978; accepted: August 28, 1978 